\title{
Multiscale Self-Assembly of Silicon Quantum Dots into an Anisotropic Three-Dimensional Random Network
}

Serim Ilday,,$^{* \dagger,}$ F. Ömer Ilday, ${ }^{\ddagger} \S$ René Hübner, $\|$ Ty J. Prosa, ${ }^{\perp}$ Isabelle Martin, ${ }^{\perp}$ Gizem Nogay, ${ }^{\#}$ Ismail Kabacelik, ${ }^{\nabla}$ Zoltan Mics, ${ }^{\bigcirc}$ Mischa Bonn, ${ }^{\bigcirc}$ Dmitry Turchinovich, ${ }^{\bigcirc}$ Hande Toffoli, ${ }^{\#}$ Daniele Toffoli, David Friedrich," Bernd Schmidt," Karl-Heinz Heinig," and Rasit Turan"

${ }^{\dagger}$ Department of Micro and Nanotechnology, Middle East Technical University, 06800, Ankara, Turkey

${ }^{\ddagger}$ Department of Physics and ${ }^{\S}$ Department of Electrical and Electronics Engineering, Bilkent University, 06800, Ankara, Turkey

"Institute of Ion Beam Physics and Materials Research, Helmholtz-Zentrum Dresden-Rossendorf, D-01328 Dresden, Germany

${ }^{\perp}$ CAMECA Instruments Inc., Madison, Wisconsin 53711 United States

\#Department of Physics, Middle East Technical University, 06800, Ankara, Turkey

${ }^{\nabla}$ Department of Physics, Akdeniz University, 07058, Antalya, Turkey

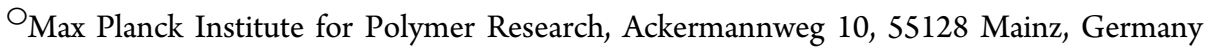

Dipartimento di Scienze Chimiche e Farmaceutiche, Universita di Trieste, Via L. Giorgieri 1, 34127 Trieste, Italy

\section{Supporting Information}

ABSTRACT: Multiscale self-assembly is ubiquitous in nature but its deliberate use to synthesize multifunctional three-dimensional materials remains rare, partly due to the notoriously difficult problem of controlling topology from atomic to macroscopic scales to obtain intended material properties. Here, we propose a simple, modular, noncolloidal methodology that is based on exploiting universality in stochastic growth dynamics and driving the growth process under far-from-equilibrium conditions toward a preplanned structure. As proof of principle, we demonstrate a confined-butconnected solid structure, comprising an anisotropic random network of silicon quantum-dots that hierarchically self-assembles from the atomic to the microscopic scales. First, quantum-dots form to subsequently interconnect without inflating their diameters to form a random network, and this network then grows in a preferential direction to form undulated

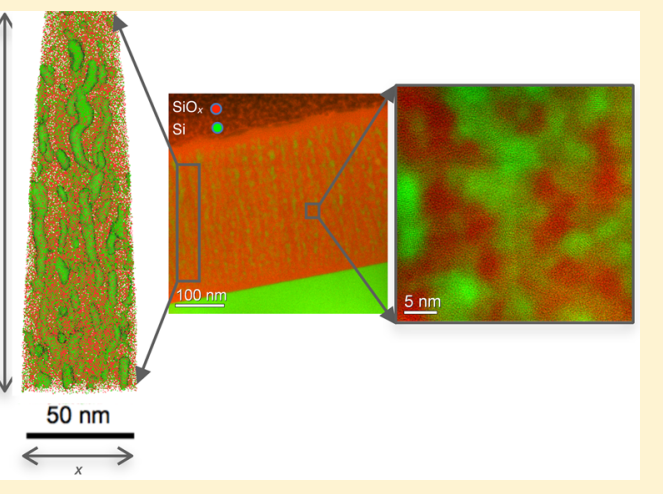
and branching nanowire-like structures. This specific topology simultaneously achieves two scale-dependent features, which were previously thought to be mutually exclusive: good electrical conduction on the microscale and a bandgap tunable over a range of energies on the nanoscale.

KEYWORDS: Si, random network, hierarchical, multiscale, self-assembly, stochastic deposition

$\mathrm{O}$ ne of the well-known challenges in the design of nanomaterials is to simultaneously achieve material properties originating from few-atom scale and resulting in bulk properties through which the material connects to other materials or interacts with devices. ${ }^{1-3}$ This is difficult because properties arising from physics at different scales are often mutually exclusive., ${ }^{4,5}$ Topology and material properties are intimately coupled and certain topologies with higher complexity, such as interconnected networks of low-dimensional quantum-confined structures, can give rise to fundamentally different material characteristics. ${ }^{1,3-8}$ They can even exhibit completely new features, which are absent in their individual constituents, but emerge from their collective interactions as defined in part by their topology. ${ }^{1,45,9,10}$ However, it is rarely clear a priori how such a topology should look like in order to solve a given problem and typically it is even more difficult to fabricate that particular topology. Top-down approaches require numerous complex steps and high-precision control over virtually all degrees of freedom for fabrication of such multiscale, multifunctional materials. These factors render fabrication techniques not only complex but also highly specific to the particular material system, which limit compatibility with well-established processing technologies. Self-assembly is an elegant and potentially simpler alternative, ${ }^{1,5,9,11,12}$ but directing the self-assembly process toward a preplanned structure is also notoriously difficult and there is no systematic approach to determine the experimental procedure for self-assembly of a given structure. Consequently, self-assembly based solutions also tend to be highly material specific.

Received: December 17, 2015

Revised: February 8, 2016

Published: February 11, 2016 

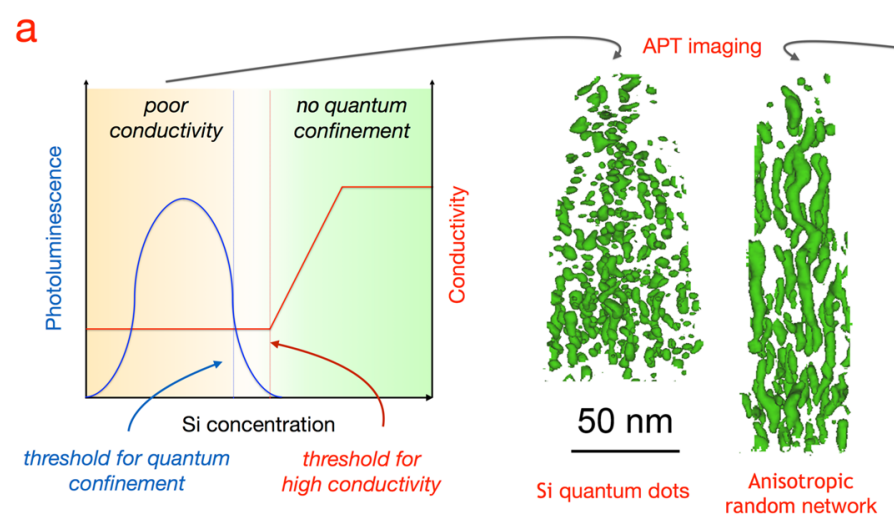

b
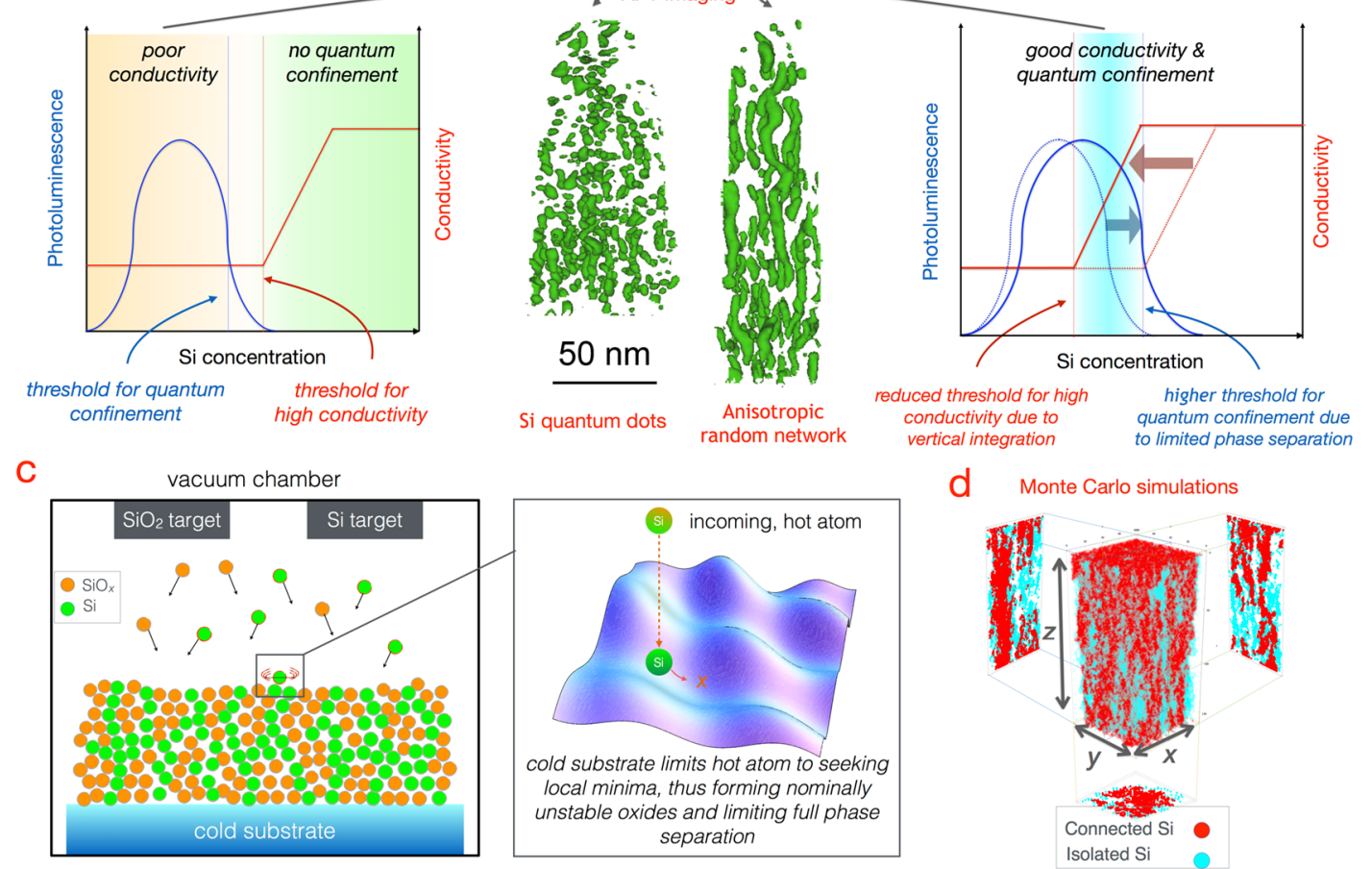

Figure 1. Conceptual methodology. Sketches of (a) dependence of photoluminescence and electrical conductivity on Si concentration. For regular nanocrystals, Si concentration thresholds of quantum confinement and good conductivity do not overlap. (b) For the random network, a region of a confined-but-connected structure emerges upon pushing and pulling the two thresholds toward each other through promoting vertical growth of the nanocrystals and limiting full phase separation of $\mathrm{Si}$ and $\mathrm{SiO}_{2}$. Atom probe tomography (APT) images show 2D projections of the 3D images, where a random sampling of Si ions (green) is displayed for isolated QDs (for $x=1.29$ ) and anisotropic random network structure (for $x=1.07)$ on the left- and right-hand-side images, respectively. (c) Schematics of the deposition chamber. (Inset) Incoming hot atoms lose most of their kinetic energy upon collision with the cold substrate and are confined to a local energy minimum within a few nanometers of their impact points. (d) Monte Carlo simulation of microscale topology of the random network, showing its preferentially vertical alignment. Red and cyan dots indicate electrically percolated and isolated Si nanocrystals, respectively.

A prototypical and also technologically important example is the 30-year-old challenge of realizing a connected-but-confined silicon nanostructure embedded in a dielectric matrix (e.g., $\mathrm{SiO}_{2}$ ) that combines quantum-dot-like optical properties and good electrical conduction: ${ }^{6,13,14}$ These properties are seemingly contradicting because charge confinement goes hand-inhand with localization. While there is a large body of work on confined-but-connected quantum-dots, ${ }^{15,10}$ this has not been demonstrated in a Si system except for the demonstration of quantum confinement effects above the percolation threshold. ${ }^{17}$ Porous Si has also shown to exhibit confinement and connectedness, however, with poor electrical conduction and lack of control over the bandgap. ${ }^{6,13,14}$ This is so because there are major challenges from materials perspective such as control over the pore sizes and the uniformity of the pores and stability problem due to the high possibility of oxidation under atmospheric conditions. ${ }^{6,13,14}$ Moreover, an overwhelming majority of the studies on confined-but-connected materials use (functionalized) colloidal particles. ${ }^{15,16,18}$ Apart from being strongly material specific, the fabrication procedure is also complicated and requires multiple steps, that is, nanoparticle synthesis, postannealing of the nanoparticles to connect the dots, recovery of the colloidal film from the solution, and its cleaning. ${ }^{15,16}$ There is much motivation for the development of a Si-based material, because $\mathrm{Si}$ is the material of choice for optoelectronic and photovoltaic applications by virtue of its low cost, natural abundance, and excellent compatibility with wellestablished processing technologies. ${ }^{6,13}$
The origin of the difficulty of achieving quantum confinement and good electrical conductivity in a Si system is shown schematically in Figure 1a. Basically, the maximum permissible Si concentration for which quantum confinement is preserved is prohibitively low for conductivity with a purely random distribution of the nanocrystals. The limited number of $\mathrm{Si}$ atoms in contact prevents the formation of extensive current routes. Consequently, charge transport has to rely on tunneling, which is extremely inefficient. ${ }^{6,13,14}$ The straightforward remedy is to increase the Si concentration, thus forming a percolated crystal network that can provide sufficient routes for efficient charge transport. This fails since it inadvertently inflates average nanocrystal sizes beyond the critical Bohr radius $(\sim 5 \mathrm{~nm})$, resulting in loss of quantum confinement, that is, an overcrowded network. ${ }^{6,13,14}$

Here, we set out to address this problem through a tailored material topology that we aimed to create through selfassembly. We did so by using a toy model to determine stochastic growth parameters that would result in the desired topology. We then created and exploited far-from-equilibrium conditions during deposition to shape the stochastic dynamics toward achieving this preplanned topology. Namely, we have created an anisotropic random network of $\mathrm{Si}$ quantum dots (QDs). At the atomic scale, QDs are formed under nonequilibrium conditions, which we exploit to prevent full phase separation of $\mathrm{Si}$ and $\mathrm{SiO}_{2}$ through stabilizing nominally unstable suboxides $\left(\mathrm{SiO}_{x}\right)$. These QDs sparsely interconnect without inflating their diameters to form an anisotropic random 
network, thereby increasing the threshold for quantum confinement to higher $\mathrm{Si}$ concentrations. On larger scales, this network becomes anisotropic, preferentially growing in the vertical direction to form nanowire-like structures. This particular topology decreases the $\mathrm{Si}$ concentration threshold for electrical percolation (Figure $1 \mathrm{~b}$ ). Therefore, a range of $\mathrm{Si}$ concentration values emerge within which both the requirements of quantum confinement and electrical conductivity are satisfied. We report simultaneous achievement of good electrical conductivity $(\sim 0.1 \mathrm{~S} / \mathrm{m})$ and a bandgap tunable over the visible light range (from $\sim 1.8$ to $2.7 \mathrm{eV}$ ).

Because the requirements of good conductivity and quantum confinement pertain to different length scales, it was logical to seek a solution in the form of a multiscale structure. In our case, there are two well-known nanostructures that already meet our requirements, albeit one at a time. Quantum confinement requires isolated low-dimensional quantum-confined structures, like QDs (OD) and good electrical conductivity can be achieved with well-connected extended structures, like nanowires (1D). Therefore, we aimed for a multiscale topology that can be conceptualized by means of a fusion of these two types of structures.

In order to determine how to self-assemble such a topology, we developed a toy model of the stochastic deposition process (Figure 1c) from which we related the intended topology to only three stochastic growth parameters. According to this model, $\mathrm{Si}$ and $\mathrm{SiO}_{x}(x$ is the $\mathrm{O} / \mathrm{Si}$ ratio and $0<x<2)$ particles are incident on the surface with a probability of $p_{1}=1 /(1+x)$ and $1-p_{1}$, respectively. The value of $p_{1}$ simply determines the final ratio of $\mathrm{Si}$ to $\mathrm{SiO}_{x}$. Once the particles come into contact with the surface, they can seek and "stick" to a particle of the same type, the probability of which is related to $p_{2}$, or they can "diffuse" regardless of which type of particle they end up touching with a probability of $p_{3}$. The value of $p_{2}$ relates to the propensity of the structure to grow in straight vertical lines in a similar fashion to nanowires. Finally, $p_{3}$ describes the tendency to branch off. These three probabilities are sufficient to describe a rich variety of topologies (Figure S1), including the structure reported here (Figure 1d). Although a toy model such as this certainly cannot be used for quantitatively accurate modeling, this simplicity is crucial to easily determine the basic requirements to achieve the desired topology through Monte Carlo simulations and then to relate those requirements to experimental conditions. Higher values of $p_{2}$ lead to preferentially vertically oriented growth, meanwhile $p_{3}$ is kept low enough to preserve this orientation but not too low to avoid becoming nanowire-like, which is also undesirable. Then, it is straightforward to calculate the ratio of connected to isolated clusters of $\mathrm{Si}$ as a function of $\mathrm{Si}$ concentration (determined by $p_{1}$ ), which allows approximately $80-90 \%$ of the Si sites to be globally connected for $x=1.0-1.2$.

Having qualitatively linked the desired topology to parameters governing stochastic growth with the toy model, the next step was to achieve the corresponding experimental conditions (Figure 1c). Fabrication was performed using magnetron sputter deposition. The connection of $p_{1}$ to experiments is straightforward and is adjusted through the ratio of power applied to the $\mathrm{Si}$ and $\mathrm{SiO}_{2}$ targets during deposition. The goal is to utilize the minimum $\mathrm{Si}$ concentration that results in a percolated network, the requirement for efficient conduction. The choice of $x$ is described in detail below. The deposition parameters and $p_{2}$ and $p_{3}$ are less straightforward to implement experimentally, and these probabilities have to be regarded as effective values providing a simplified description of much more complicated dynamics affected by various experimental parameters. Nevertheless, it is not difficult to identify effective "knobs" for each of them. The sticking or affinity of the $\mathrm{Si}$ atoms to $\mathrm{Si}$ atoms is present during deposition but occurs also following a postannealing procedure, as evidenced by the fact that the vertical orientation of the network becomes more prominent after postannealing. Thus, the postannealing process parameters influence propensity to grow vertically, hence $p_{2}$. Similarly, lateral diffusion of the deposited atoms and, effectively, the value of $p_{3}$ is most readily controlled by the kinetic energy of the particles after hitting the surface and the temperature of the surface. To limit this process, we create a temperature gradient by keeping the substrate "cold" (at room temperature), while depositing "hot" particles, which are energized by the magnetrons (inset of Figure 1c). The kinetic energies of the deposited particles are controlled through magnetron power levels. Following identification of these basic experimental control knobs, it was necessary to pursue a laborious but straightforward experimental procedure iterating between fabrication runs and characterization in order to pin down the final set of deposition parameters until a structure that matches the design was obtained.

There is a second purpose for the spatiotemporal temperature gradient on the surface, which is to serve as leverage to meet our second goal of increasing $\mathrm{Si}$ concentrations for which quantum confinement can be maintained. During fast stochastic deposition, the atoms rain down onto the cold substrate, which forces the adatoms to settle at minimum energy positions within a few nanometers of the collision points, instead of seeking out the global minimum over larger portions of the surface. This way, $\mathrm{Si}$ and $\mathrm{O}$ atoms are trapped into a random network that is being braided during deposition. Even when a calibrated amount of external energy is introduced into the system (during postannealing to promote crystallization at high temperatures), the atoms are not able to move freely to form stable $\mathrm{Si}$ and $\mathrm{SiO}_{2}$ but they are forced to locally rearrange themselves that results in locking the unstable suboxides into their nonglobal but local equilibrium states and prevent full phase separation (Figures S2 and S3).

Normally, when sufficient energy is applied to the system, suboxides break down and turn into stable forms of either Si or $\mathrm{SiO}_{2}$ through a disproportionation reaction. The thermal gradient created by the cold substrate serves to stabilize suboxides, which, in turn push up the Si concentration limit for quantum confinement. If too many suboxides turn into $\mathrm{Si}$, they inflate the effective QD diameter, which eventually destroys the quantum confinement, resulting in an overcrowded network. In the other extreme, if too many suboxides turn into $\mathrm{SiO}_{2}$, they surround and isolate the QDs, which once again have to rely on inefficient tunneling currents for conduction. The key issue then is to obtain nominally unstable suboxides and to prevent them from turning into stable forms of $\mathrm{Si}$ and $\mathrm{SiO}_{2}$ completely upon addition of external energy, thereby limiting further growth of $\mathrm{QD}$ diameters. Creation of far-from-equilibrium conditions, as opposed to merely near-equilibrium, via large spatiotemporal thermal gradients is important in stabilizing the suboxides. This way, a larger number of small-size QDs are obtained instead of a smaller number of large-size QDs in a given volume, which more readily form a percolated network due to increased density of connections. 
Having discussed the methodology of the design and selfassembly, we first present characterization of the multiscale topology of the network, followed by its electrical and optical properties. The topology of the Si QD network was designed to be anisotropic on the microscale (over tens to hundreds of nanometers) and isotropic on the nanoscale (up to $\sim 10 \mathrm{~nm}$ ). This is verified experimentally through atom probe tomography (APT) and energy-filtered transmission electron microscopy (EFTEM). Figure $2 \mathrm{a}$ shows $2 \mathrm{D}$ projection of a 3D APT image

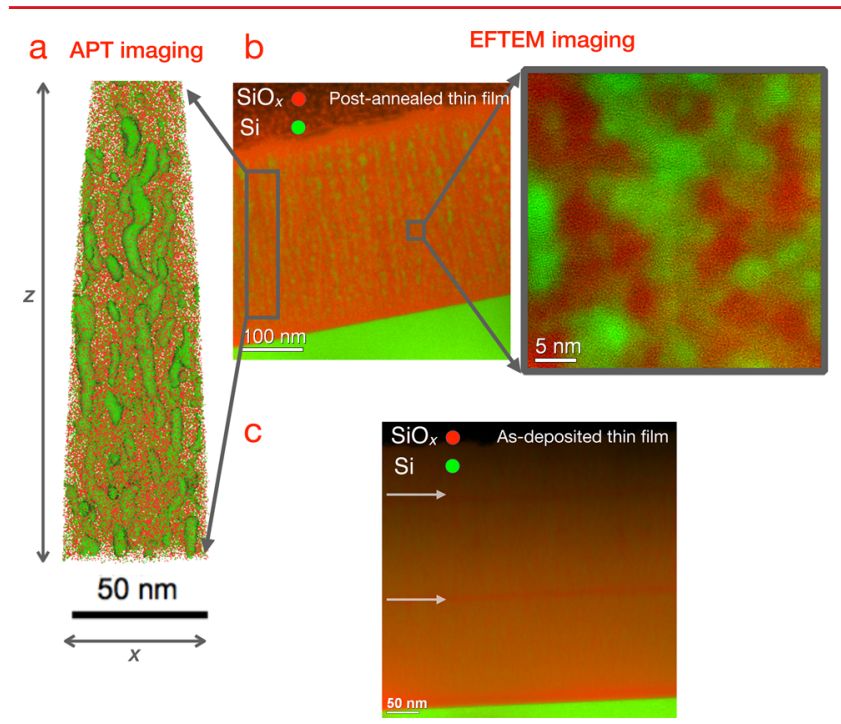

Figure 2. Structural and morphological analyses. (a) The 2D projections of 3D APT images, where a random sampling of the $\mathrm{Si}$ ions (green) and $\mathrm{O}$ ions (red) are displayed for anisotropic random network structure (for $x=1.07$ ). Superposition of the $\mathrm{Si}$ (green) and the $\mathrm{SiO}_{x}$ (red) plasmon EFTEM images showing (b) preferential growth of the network along the vertical direction on the microscale and (inset) percolated $\mathrm{Si} \mathrm{QD}$ network inside a $\mathrm{SiO}_{x}$ matrix on the nanoscale for anisotropic random network structure (for $x=1.07$ ) and (c) vertical orientation of $\mathrm{Si}$ atoms before any postannealing procedure is applied (for $x=1.07$ ).

of the random network, where a random sampling of the Si ions (green) and O ions (red) is displayed (see also the Supporting Movie). The APT analysis establishes the vertical orientation unambiguously, which is also discernible from the EFTEM images. Figure $2 \mathrm{~b}$ shows pseudocolored superposition of the Si (green) and $\mathrm{SiO}_{x}$ (red) EFTEM image of the random network of $\mathrm{Si}$ QDs that is oriented preferentially in the vertical direction resembling nanowires and the magnified image displays interconnected Si QDs with $2 \mathrm{~nm}$ average diameters (Figure S4) that are embedded inside the $\mathrm{SiO}_{x}$ matrix.

The EFTEM and APT data suggest that many of the Si QDs are in physical contact with each other without any $\mathrm{SiO}_{x}$ between them, as predicted by the Monte Carlo simulations. This has profound implications for charge transport; charge can be transported directly without relying on tunneling. In order to ascertain that this is the case, we have applied a tomography technique, whereby spatial distributions of $\mathrm{Si}$ QDs and the surrounding amorphous $\mathrm{SiO}_{x}$ matrix were determined separately by energy filtering analysis (Figure S5). Additional diffraction analysis was used to prove that there are distinct $\mathrm{Si}$ nanocrystals, which are directly touching each other without any $\mathrm{SiO}_{x}$ in between.

We have mentioned above that the vertical orientation of the network becomes more pronounced after postannealing as this process triggers a limited degree of Si mobility and tendency to stick together. In order to rule out the possibility that the preferential vertical growth could be solely the result of a postannealing procedure, a test specimen has been prepared and was analyzed by EFTEM directly after magnetron sputter deposition without any postannealing procedure as shown in Figure 2c. During deposition of this test sample, sputtering from the Si target was interrupted twice to ensure that the directional growth could reinitiate, as would be expected, considering that the growth is stochastic and obeys the rules we have predetermined to control surface diffusion. As seen in Figure $2 c$, two thin red layers comprising only suboxides (areas pointed by two arrows) appear after which the vertical growth of $\mathrm{Si}$ has continued in the same manner. We further observe that the network is already anisotropic (albeit less pronounced) even though no postannealing was performed on this sample.

The desired large-scale anisotropy of the QD network topology is achieved for a range of Si concentrations, which we refer to as the network region. Si concentrations that delimit this interesting region from below and above are similarly referred to as the "prenetwork" and "postnetwork" regions. Within this range, the average QD diameters can be controlled through adjustment of the Si concentration. This is confirmed through EFTEM images shown in Figure 3. Nanocrystal diameters increase and the morphology becomes more wire-like when $\mathrm{Si}$ concentration is increased (when $x$ decreases from 1.07 to 0.95$)$.

Figure 4a shows a typical magnified APT image of the random network. The image reveals that although the network structure somewhat resembles nanowires on the microscale, the vertical lines are far from being straight and they can be referred
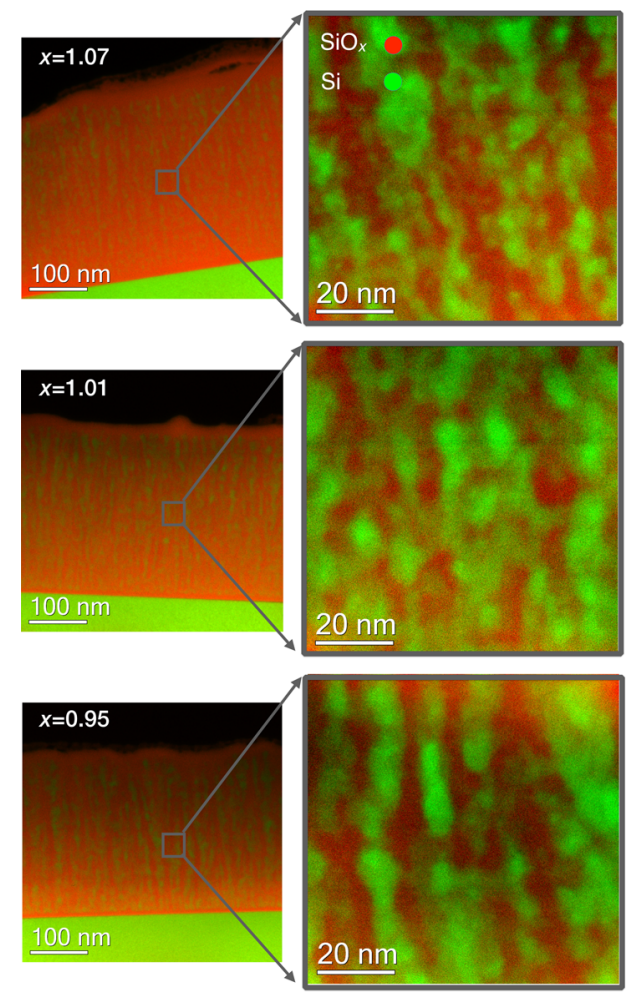

Figure 3. Superposition of the cross-sectional $\mathrm{Si}$ (green) and $\mathrm{SiO}_{x}$ (red) plasmon EFTEM images show how QD diameters in the random network structure changes with respect to increased $\mathrm{Si}$ concentration (from $x=1.07$ to $x=0.95$ ). 

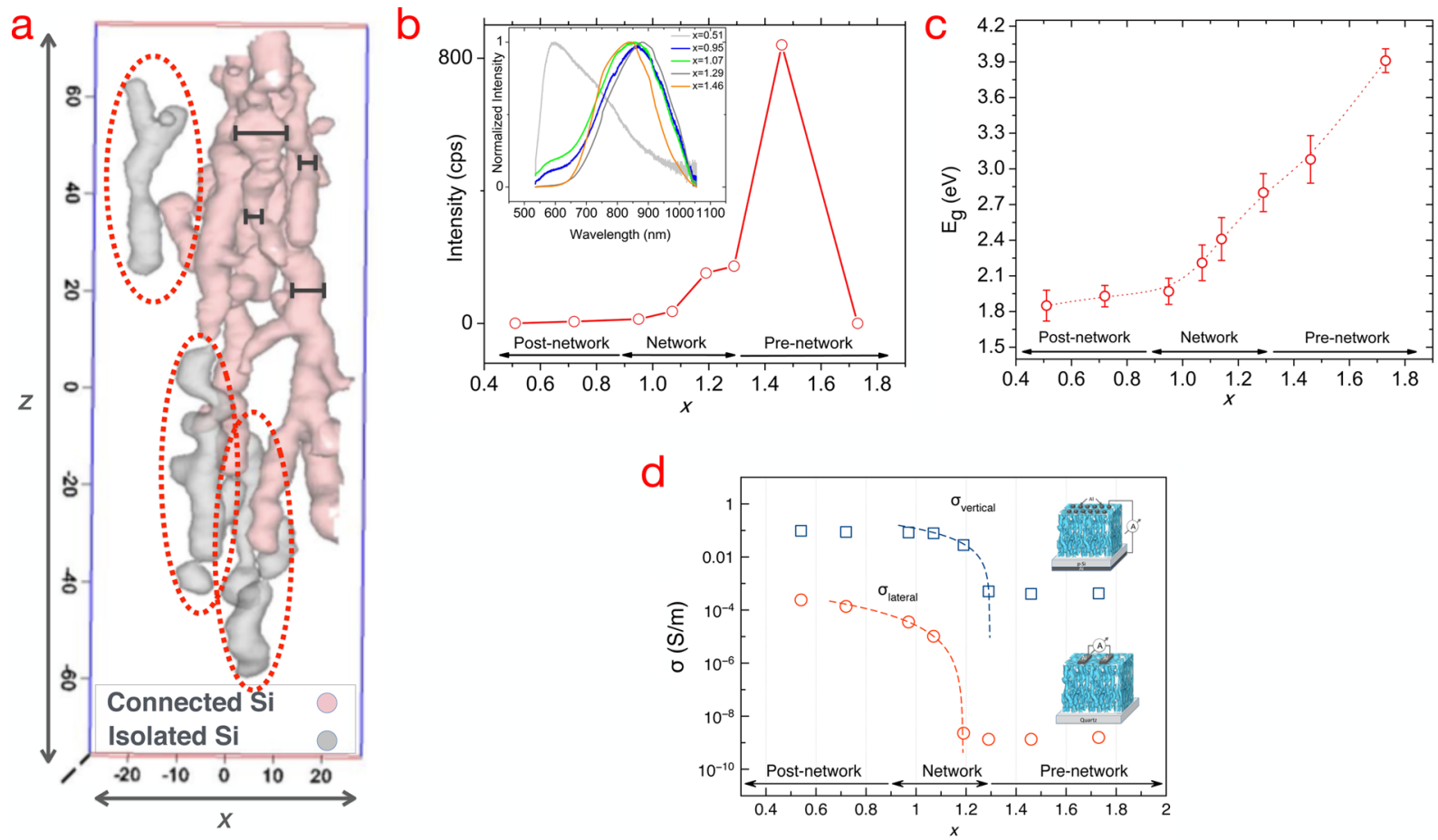

Figure 4. Quantum confinement and percolation analyses. (a) APT image shows undulated and branching wirelike morphology of the random network (for $x=1.07$ ) where the wire diameter differentiate spatially. The externally connected $\mathrm{Si}$ QD cluster is colored pink and internally connected but externally isolated clusters are colored gray (circled in dash lines). (b) Graph showing a highly intense PL signal for the prenetwork region, a reduced PL signal intensity for the network region, and virtually zero PL signal for the postnetwork region. (Inset) Graph showing full PL spectra for the three regions. (c) Graph showing tunable bandgap from prenetwork to network and postnetwork regions. (d) Graph showing percolation analyses and electrical conductivity in the lateral and vertical directions for prenetwork, network, and postnetwork regions. Inset figures showing cartoons of the devices used to conduct lateral (bottom image) and vertical (top image) $I-V$ measurements.

to as "wires" only as a crude approximation. They are rather heavily undulated, frequently interconnecting and branching. The effective wire diameters vary spatially as pointed out by markings in Figure 4a. This specific topology has implications for charge transport and photoemission with subtly different characteristics for charges that are locally generated through the photoelectric effect and for those that are injected externally through the electrodes (see Supporting Information).

Photoelectrons are generated only at sites where a photon is absorbed, which is more likely to occur at quantum-confined sites. Therefore, strong PL emission signal is expected for the prenetwork region comprising mostly isolated QDs. For the postnetwork region, PL signal will vanish as a result of nearly complete loss of confinement within a bulklike crystalline structure. PL signal is expected to decrease upon emergence of an interconnected random network because free charge carriers will belong to a cluster of QDs, which increases the probability of charge carrier pair separation because they are free to move to larger distances. ${ }^{8,19-21}$ This de facto decreases the probability of recombination of the charge carrier pair. ${ }^{8,19-21}$ However, thanks to the network being sparely connected, quantum confinement will be preserved on average, resulting in a less intense but still strong PL emission. These expectations are confirmed by the PL measurements as shown in Figure $4 \mathrm{~b}$.

Control of the average QD diameter in the network topology naturally affords us the important capability of tuning the optical bandgap of the random network from $\sim 1.8$ to $2.7 \mathrm{eV}$, covering most of the visible spectrum, as confirmed experimentally (Figure 4b,c). Although the bandgap values and PL emission energies are consistent and within the nominal values for quantum-confined Si nanostructures, ${ }^{6,13,22-25}$ there is a shift between the bandgap values calculated from the optical absorption (Figure 4c) and the PL emission spectra (inset of Figure $4 \mathrm{~b}$ ). Previous studies reported this phenomena to be the influence of exciton binding energy and/or Stokes-shift (depending on the material topology and nanocrystal-matrix interface) for $\mathrm{Si}$ nanostructures ${ }^{13,23-25}$ as well as for other nanomaterials. ${ }^{26-28}$

Of particular interest is the observation of percolation through direct current conductivity measurements, because percolation provides an intellectual bridge between structural information, which is a locally determined property of a network and electrical characteristics that are determined collectively by the entire network. ${ }^{29}$ Furthermore, percolation behavior is often robust against variations in many parameters or characteristics as long as the topology remains unchanged. ${ }^{29}$ Results of current-voltage $(I-V)$ conductivity measurements are shown in Figure 4d. As expected, when the Si concentration is increased, conductivity increases abruptly after a critical value of $x$ in both lateral and vertical directions is exceeded, which corresponds to a $\mathrm{Si}$ concentration below the nominal percolation threshold $\left(x_{c}=\sim 1\right)$ of $\mathrm{Si}$ in a $\mathrm{SiO}_{x}$ matrix. ${ }^{8,19,29,30}$ Conductivity values approaching the percolation threshold, $x_{\mathcal{O}}$ from above were fit by $\sigma \sim\left(x_{c}-x\right)^{t}$, where $t$ is the critical percolation exponent. ${ }^{8,19,29,30}$ The estimated values of $x_{\mathrm{c}}=1.29$ and $t=2.0$ for vertical conductivity, and $x_{\mathrm{c}}=1.18$ and $t=1.3$ for lateral conductivity. The observation of a lower percolation threshold for the vertical direction than the lateral direction is an independent proof of the anisotropic structure. We note that the critical exponents for vertical and lateral conductivity 
closely match the universal values for $3 \mathrm{D}$ and $2 \mathrm{D}$ conduction networks, respectively. ${ }^{8,19,29,30}$ This direction-dependent dimensionality is remarkable, but not surprising, given the topology of the network. For conductivity in the lateral direction, as additional conducting nodes are added (decreasing $x$ ), few of those that are along the vertical direction contribute to conductivity along the lateral direction, because many of them efficiently lead above or below the lateral plane. Consequently, the percolation characteristics are expected to be closer to that of a nominally $2 \mathrm{D}$ network. In other words, the anisotropy effectively reduces the dimensionality of the network for conductivity along this direction. In contrast, the percolation behavior along the vertical direction better matches that of a 3D network, because additions of conducting nodes contribute to conduction along the vertical direction similarly.

In conclusion, we propose a simple methodology based on exploiting universality in stochastic growth dynamics and use of physical forces, such as thermal gradients and control of chemical interactions, to achieve self-assembly of a preplanned structure. We applied our approach to the design and fabrication of a nanostructured Si-based material, which brings together QD-like optical properties and good electrical conduction as Priolo et al. envisioned ${ }^{6}$ with possible applications in $\mathrm{Si}$ photonics, ${ }^{6,8,31,32}$ photovoltaics, ${ }^{6-8,14,15,31,32}$ and optoelectronics. ${ }^{6-8,31,32}$ Our design procedure utilized a toy model that links desired topology to effective stochastic growth parameters for the self-assembly process. We have qualitatively related the growth parameters to the experimental parameters with the help of the model and determined their specific values experimentally. Given the simplicity of this methodology, it is reasonable to expect that the present approach of utilizing universality of stochastic growth dynamics toward creation of a multiscale topology can be adapted to different material systems that can benefit from multiscale selfassembled structures. Experimentally, the resulting structure is not only robust after exposure to air for more than a year but also survives prolonged annealing at high temperatures. Furthermore, it is highly uniform within a sample and reproducible from sample to sample. We have characterized its optical and electrical responses, which provide an independent confirmation of the multiscale topology. In particular, the latter has revealed that motion of the charge is microscopically anisotropic but nanoscopically isotropic. Apart from a number of possible photonic and optoelectronic applications, our findings suggest that this complex random network may also be used in biophotonics, ${ }^{32}$ imaging technologies, $^{32}$ Li-ion batteries, ${ }^{32,33}$ and chemical and biomedical sensors. ${ }^{34}$

\section{ASSOCIATED CONTENT}

\section{S Supporting Information}

The Supporting Information is available free of charge on the ACS Publications website at DOI: 10.1021/acs.nanolett.5b05158.

Methods, detailed calculations and simulations, and detailed information on the chemical, structural, optical. and electrical analyses. (PDF)

APT image of random network where random sampling of the $\mathrm{Si}$ ions (green) and $\mathrm{O}$ ions (red) is displayed. (AVI)

\section{AUTHOR INFORMATION}

\section{Corresponding Author}

*E-mail: serim@bilkent.edu.tr.

\section{Author Contributions}

S.I. conceived of and conducted the experiments. S.I. and F.Ö.I. developed the stochastic deposition model and wrote the paper. F.Ö.I. performed the MC simulations. R.H. conducted TEM, T.J.P,. and I.M. conducted APT imaging and analyses. S.I., G.N., and I.K. performed electrical analyses. Z.M., D.T., and M.B. conducted $\mathrm{THz}$ spectroscopy analyses. H.U. and D.T. conducted MD simulations. D.F. conducted RBS analyses and assisted in TEM imaging. B.S. and K.H.H. supervised TEM imaging and RBS analyses. R.T. supervised the study.

\section{Funding}

This work was supported by the Scientific and Technological Research Council of Turkey (TÜBİTAK) and the German Federal Ministry of Education and Research (BMBF) with Grant 109R037. F.Ö.I. also acknowledges support from the European Research Council (ERC) Consolidator Grant ERC617521 NLL.

\section{Notes}

The authors declare no competing financial interest.

\section{ACKNOWLEDGMENTS}

The authors thank to İlker Yıldız and Seçkin Öztürk for their contributions to XPS and early HRTEM analyses, respectively, and to Alexandre Houssou and Dr. Olivier Dulac for their contributions in APT analyses.

\section{REFERENCES}

(1) Boal, A. K.; Ilhan, F.; DeRouchey, J. E.; Thurn-Albrecht, T.; Russell, T. P.; Rotello, V. M. Nature 2000, 404, 746-748.

(2) Lopinski, G. P.; Wayner, D. D. M.; Wolkow, R. A. Nature 2000, 406, 48-51.

(3) Tavakkoli, A. K. G.; Gotrik, K. W.; Hannon, A. F.; AlexanderKatz, A.; Ross, C. A.; Berggren, K. K. Science 2012, 336, 1294-1298.

(4) Hammond, P. T. Adv. Mater. 2004, 16, 1271-1293.

(5) Mann, S. Nat. Mater. 2009, 8, 781-791.

(6) Priolo, F.; Gregorkiewicz, T.; Galli, M.; Krauss, T. F. Nat. Nanotechnol. 2014, 9, 19-32.

(7) Escarcega-Bobadilla, M. V.; Zelada-Guillen, G. A.; Pyrlin, S. V.; Wegrzyn, M.; Ramos, M. M. D.; Gimenez, E.; Stewart, A.; Maier, G.; Kleij, A. W. Nat. Commun. 2013, 4, 2648.

(8) Nie, Z.; Petukhova, A.; Kumacheva, E. Nat. Nanotechnol. 2010, 5, $15-25$.

(9) Jia, G.; Sitt, A.; Hitin, G. B.; Hadar, I.; Bekenstein, Y.; Amit, Y.; Popov, I.; Banin, U. Nat. Mater. 2014, 13, 301-307.

(10) Damasceno, P. F.; Engel, M.; Glotzer, S. C. Science 2012, 337, 453-457.

(11) Whitesides, G. M.; Grzybowski, B. Science 2002, 295, 24182421.

(12) Zhao, Y.; Thorkelsson, K.; Mastroianni, A. J.; Schilling, T.; Luther, J. M.; Rancatore, B. J.; Matsunaga, K.; Jinnai, H.; Wu, Y.; Poulsen, D.; Fréchet, J. M. J.; Alivisatos, A. P.; Xu, T. Nat. Mater. 2009, 8, 979-985.

(13) Pavesi, L.; Turan, R. Si Nanocrystals: Fundamentals, Synthesis, and Applications; Wiley-VCH Verlag GmbH \& Co.: Berlin, 2010.

(14) Balberg, I.; Jedrzejewski, J.; Savir, E. Phys. Rev. B: Condens. Matter Mater. Phys. 2011, 83, 035318.

(15) Baumgardner, W. J.; Whitham, K.; Hanrath, T. Nano Lett. 2013, 13, 3225 .

(16) Evers, W. H.; Schins, J. M.; Aerts, M.; Kulkarni, A.; Capiod, P.; Berthe, M.; Grandidier, B.; Delerue, C.; van der Zant, H. S. J.; van Overbeek, C.; Peters, J. L.; Vanmaekelbergh, D.; Siebbeles, L. D. A. Nat. Commun. 2015, 6, 8195. 
(17) Wolf, O.; Millo, O.; Balberg, I. J. Appl. Phys. 2013, 113, 144314.

(18) Milliron, D. J.; Hughes, S. M.; Cui, Y.; Manna, L.; Li, J.; Wang, L.-W.; Alivisatos, A. P. Nature 2004, 430, 190-195.

(19) Balberg, I.; Savir, E.; Jedrzejewski, J.; Nassiopoulou, A. G.; Gardelis, S. Phys. Rev. B: Condens. Matter Mater. Phys. 2007, 75, 235329.

(20) Cox, T. I.; Simons, A. J.; Loni, A.; Calcott, P. D. J.; Canham, L. T.; Uren, M. J.; Nash, K. J. J. Appl. Phys. 1999, 86 (5), 2764-2773.

(21) Keles, U.; Cakan, A.; Bulutay, C. J. Appl. Phys. 2015, 117, 064308.

(22) Tomozeiu, N. Optoelectronics-Materials and Techniques; InTech: Rijeka, Croatia, 2011; Chapter 3.

(23) Pavesi, L.; Gaponenko, S.; Dal Negro, L. Towards the First Silicon Laser; Springer: Trento, Italy, 2002.

(24) Di, D.; Xu, H.; Perez-Wurfl, I.; Green, M. A.; Conibeer, G. Nanoscale Res. Lett. 2011, 6, 612.

(25) Le, T.-H.; Jeong, H.-D. Bull. Korean Chem. Soc. 2014, 35, 1523.

(26) Prasad, A. V.; Misra, P.; Ahirwar, G. Res. J. Phys. Sci. 2013, 1, $11-14$.

(27) So, D.; Konstantatos, G. Chem. Mater. 2015, 27, 8424-8432.

(28) Ahmad, S.; Baumberg, J. J.; Prakash, G. V. J. Appl. Phys. 2013, $114,233511$.

(29) Stauffer, D.; Aharony, A. Introduction to Percolation Theory; Taylor \& Francis: London, 1992.

(30) Manna, L.; Milliron, D. J.; Meisel, A.; Scher, E. C.; Alivisatos, A. P. Nat. Mater. 2003, 2, 382-385.

(31) Gudiksen, M. S.; Lauhon, L. J.; Wang, J.; Smith, D. C.; Lieber, C. M. Nature 2002, 415, 617-620.

(32) Dasgupta, N. P.; Sun, J.; Liu, C.; Brittman, S.; Andrews, S. C.; Lim, J.; Gao, H.; Yan, R.; Yang, P. Adv. Mater. 2014, 26, 2137-2184. (33) Bogart, T. D.; Oka, D.; Lu, X.; Gu, M.; Wang, C.; Korgel, B. A. ACS Nano 2014, 8, 915-922.

(34) Cui, Y.; Wei, Q.; Park, H.; Lieber, C. M. Science 2001, 293, $1289-1292$. 